\title{
NIVELES DE LECTURA Y GONTEXTOS EN LAS ACTIVIDADES SOBRE TABLAS ESTADÍSTICAS EN LIBROS DE TEXTO GHILENOS Y ESPAÑOLES
}

\author{
READING LEVELS AND CONTEXT IN ACTIVITIES ON STATISTICAL TABLES \\ IN CHILEAN AND SPANISH TEXTBOOKS
}

Jocelyn D. Pallauta

jocelyndiaz@correo.ugr.es

Universidad de Granada,

Granada, España

\author{
Carmen Batanero \\ batanero@ugr.es \\ Universidad de Granada, \\ Granada, España
}

\author{
María M. Gea \\ mmgea@ugr.es \\ Universidad de Granada, \\ Granada, España
}

\author{
Pedro Arteaga \\ parteaga@ugr.es \\ Universidad de Granada, \\ Granada, España
}

\begin{abstract}
RESUMEN
Las tablas estadísticas son frecuentemente utilizadas para presentar y analizar la información, lo que lleva a incluir su enseñanza en la educación obligatoria. En este trabajo se analizan las actividades planteadas en torno a las tablas estadísticas en una muestra de libros de texto chilenos y españoles, dirigidos a los niveles $7^{\circ}$ y $8^{\circ}$ básico chilenos ( $1^{\circ}$ y $2^{\circ}$ de secundaria españoles). Las actividades se clasifican según sus niveles de lectura. También se analiza el contexto de las tareas, siguiendo los propuestos en los estudios PISA, en los que, adicionalmente, se incorporan las categorías de experimentos aleatorios y actividades sin contexto. Los resultados muestran un ligero incremento hacia los niveles sofisticados de lectura, conforme se progresa de curso, tanto en los libros chilenos como españoles, mientras que el contexto que aparece con gran fuerza en la muestra es el de tipo personal.
\end{abstract}

PALABRAS CLAVE:

Libros de texto; tablas estadísticas; niveles de lectura; contexto.

\begin{abstract}
Statistical tables are frequently used to present and analyze information and are therefore included in compulsory education. In this paper, we analyze the activities proposed on statistical tables in a sample of Chilean and Spanish textbooks directed to 7th and 8th grades in Chile (1st and 2nd levels of Spanish secondary school). The activities are classified according to their reading levels. The context of the tasks is also analyzed, following those proposed in the PISA studies, to which random experiments and activities without context are added. The results show a slight increase towards the s'mores sophisticated levels of reading, as the course progresses, both in the Chilean and Spanish textbooks, while the most frequent context in the sample is the personal context.
\end{abstract}

\section{KEYWORDS:}

Textbooks; statistical tables; reading levels;

context. 


\section{Introducción}

En los medios de comunicación, así como en el trabajo profesional, se encuentran con frecuencia tablas estadísticas que cualquier ciudadano competente necesita interpretar para comprender su información y tomar buenas decisiones, especialmente en el contexto de incertidumbre que la sociedad ha debido enfrentar por la pandemia generada por la COVID-19 (RodríguezMuñiz et al., 2020). Por este motivo, la competencia con las tablas estadísticas la entendemos como parte de la alfabetización estadística, o capacidad para comprender e interpretar la información estadística y razonar sobre ella (Gal, 2019; Gould, 2017).

Este trabajo se centra en los libros de texto, que constituyen un recurso didáctico valioso, al ser un puente entre las directrices curriculares y el trabajo del aula (Herbel, 2007). Esta importancia se ve reflejada en el aumento de la investigación sobre el libro de texto de matemáticas (Pepin y Gueudet, 2020), ya que se ha mostrado que este recurso tiene una influencia directa en el aprendizaje del estudiante (Reys et al., 2004).

El estudio de la tabla estadística es importante no solo por su relevancia en la presentación de la información en diferentes áreas del conocimiento, sino también debido a que su comprensión es básica para el aprendizaje de otros temas de la estadística (Estrella y Estrella, 2020). Su enseñanza se incluye en Chile desde los primeros cursos de educación básica (Ministerio de Educación de Chile [MINEDUC], 2018): de $1^{\circ}$ a $3^{\circ}$ básico se propone el trabajo con tablas de conteo, como un medio para organizar datos que pueden emanar del entorno cercano al estudiante. A partir de $4^{\circ}$ básico se incluyen las tablas con frecuencias absolutas para registrar, entre otros, los resultados obtenidos de experimentos aleatorios. En $5^{\circ}$ aparecen las tablas de datos de doble entrada, mientras que en los siguientes cursos se incorporan las tablas con diferentes tipos de frecuencias (relativas, porcentuales), que son utilizadas para representar información acerca de una muestra, o para presentar los resultados de experimentos aleatorios. En $6^{\circ}$ y $7^{\circ}$ el uso de las tablas estadísticas se asocia al estudio de la probabilidad frecuencial, y en $8^{\circ}$ se hace hincapié en que los estudiantes evalúen la forma en que los datos están presentados (MINEDUC, 2015).

En las directrices curriculares de España (Ministerio de Educación, Cultura y Deporte de España [MECD], 2014), a lo largo de la educación primaria (6 a 12 años) se trabaja con tablas estadística para la recogida y clasificación de datos cualitativos y cuantitativos, así como la construcción de tablas de frecuencias absolutas y relativas. En la enseñanza secundaria (MECD, 2015), en los dos primeros cursos (12 a 14 años) se profundiza en el estudio de las tablas con frecuencias absolutas y relativas, que se usan junto a las medidas de centralización y el rango para el estudio de la probabilidad desde el enfoque frecuencial.
Basándonos en la relevancia de la tabla estadística en los lineamientos curriculares de ambos países, y la escasa literatura al respecto (Estrella y Estrella, 2020), nos planteamos como pregunta de investigación si influye el contexto educativo del país en el tratamiento del nivel de lectura y el contexto con que se plantea el estudio de la tabla estadística. Para responder a esta cuestión, en el presente trabajo se analizan las actividades planteadas en torno a las tablas estadísticas en una muestra de libros de texto chilenos y españoles en los niveles $7^{\circ}$ y $8^{\circ}$ básico chilenos (10 y $2^{\circ}$ de secundaria españoles), considerando las variables niveles de lectura y el contexto en que es planteada la tabla estadística, lo que nos permitirá comparar estas variables en dos contextos educativos diferentes.

En los siguientes apartados se describen los fundamentos y antecedentes que sustentan el presente trabajo, posteriormente se detalla la metodología utilizada, así como los resultados del estudio junto a las principales conclusiones.

\section{Fundamentos}

El trabajo se apoya en los niveles de lectura descritos para los gráficos y tablas estadísticas por Curcio y sus colaboradores (Friel, et al., 2001), así como en el papel del contexto en el desarrollo de la actividad matemática y también en algunos antecedentes.

\subsection{Niveles de lectura de gráficos y tablas estadísticas}

La tabla estadística es un objeto semiótico complejo (Pallauta y Arteaga, 2021), por lo que a partir de ella pueden plantearse preguntas de diferente dificultad respecto a la información que recoge. En este sentido, en el presente trabajo se utilizan los niveles de lectura establecidos por Curcio (1989) para gráficos estadísticos y ampliados luego por Shaughnessy et al. (1996) con un cuarto nivel, que finalmente fue integrado por Friel et al. (2001). Estos niveles son aplicables y también se han utilizado para analizar tablas estadísticas (Díaz-Levicoy et al., 2018; GarcíaGarcía et al., 2019; Pallauta et al., 2021). Se describen resumidamente a continuación estos niveles, que se detallarán en la sección de resultados, junto con ejemplos de su uso en las actividades analizadas.

- $\quad$ Nivel 1. Leer los datos, consiste en la lectura literal de la información expuesta en un gráfico o tabla, y corresponde al nivel más básico de comprensión. Este nivel implica identificar aspectos elementales de la tabla, con el objeto de responder a preguntas en que la información esté expuesta de manera explícita en ella, por ejemplo, la lectura del título o de una celda de la tabla.

- $\quad$ Nivel 2. Leer dentro de los datos, cuando para responder a alguna cuestión, además de obtener la información anterior, es necesario realizar operaciones aritméticas, comparaciones con los 
datos expuestos en la tabla, o encontrar relaciones entre los datos. Por ejemplo, determinar la moda en una tabla de distribución o calcular el total que corresponde a un rango de valores en la tabla.

- $\quad$ Nivel 3. Leer más allá de los datos, involucra una lectura de información que no viene directamente representada en la tabla, mediante inferencias o razonamientos sobre los datos. Para ello se requiere extrapolar, predecir o inferir a partir de los datos presentes en la tabla. Por ejemplo, si la variable es numérica, extrapolar la frecuencia de un valor no representado, o en el caso de una tabla de doble entrada, determinar si existe asociación entre las variables representadas.

- Nivel 4. Leer detrás de los datos, es realizar una valoración crítica de las conclusiones, o de la recogida y tratamiento de la información expuesta en una tabla o gráfico. Por ejemplo, juzgar la fiabilidad de la muestra o la posible manipulación de los datos. En este nivel se requiere un conocimiento del contexto en que se recogieron los datos presentados.

\subsection{El contexto en matemáticas y estadística}

La contextualización del conocimiento matemático tiene un rol importante en la manera en que el significado de cada objeto matemático es construido, aprendido, activado y transformado (Font, 2007). El contexto tiene una fuerte influencia en la comprensión de un problema y la elección de un método para su solución, pero también este puede generar dificultades para caracterizar un problema matemático (Wijaya et al., 2014).

Actualmente existe un interés creciente por la importancia del contexto en educación matemática, puesto que la comprensión y resolución de problemas contextualizados forma parte de la cultura científica, es decir, del conjunto de conocimientos y competencias que necesita el ciudadano para desenvolverse en situaciones en que requiere del conocimiento científico (Rosales et al., 2020). Los contextos atraen la atención de los estudiantes y conectan lo que se aprende en la escuela con la sociedad externa a la misma, motivándole para seguir aprendiendo (Sanmartí y Márquez, 2017).

Diversas investigaciones han puesto en evidencia la importancia de proponer a los estudiantes tareas matemáticas que reflejen la realidad (Van den HeuvelPanhuizen, 2005), es decir, situaciones que presenten aspectos del diario vivir, pues a pesar de ser de entorno cercano al aprendiz se pueden manifestar dificultades en su resolución (Blum et al., 2007; Wijaya et al., 2014). Es así como los estudios internacionales de evaluación PISA (Organización para la Cooperación y el Desarrollo Económicos [OECD], 2019) recogen este aspecto, pues se considera que la cultura matemática influye en la capacidad de formular y usar la matemática en una variedad de contextos. Los ítems matemáticos propuestos en dichas pruebas utilizan situaciones del mundo real, incluso cuando sean hipotéticas (Turner, 2006), que se clasifican en la forma siguiente:

- Contexto personal. Se trata de contextos cercanos a la experiencia del estudiante, sobre temáticas cotidianas de su entorno inmediato.

- Contexto laboral. Se presentan situaciones relacionadas con el mundo laboral y profesional en diversos ámbitos.

- Contexto público. Se trata de situaciones en el entorno local o en contextos más amplios, a las que es posible acceder a través de los diferentes medios de comunicación.

- Contexto científico. Incluye aplicaciones de las matemáticas más abstractas y complejas que los anteriores contextos, asociadas a procesos científicos o tecnológicos.

En este documento, se señala que el contexto moviliza diversos procesos cognitivos en el estudiante cuando resuelve un problema, por lo que en este estudio también se aborda esta variable, que ha sido valorada en algunos análisis de las actividades sobre tablas estadísticas propuestas en libros de texto (DíazLevicoy et al., 2015, 2018; García-García et al., 2019; Pallauta et al., 2021).

\subsection{Antecedentes}

La investigación cuenta con antecedentes de estudios que han analizado, entre otras variables, los niveles de lectura o los contextos de las actividades propuestas en relación a las tablas estadísticas en libros de texto dirigidos a diferentes niveles educativos.

Los principales antecedentes de análisis de tablas estadísticas en los libros de texto chilenos dirigidos a los primeros cursos de educación primaria son aportados por Díaz-Levicoy y colaboradores (DíazLevicoy et al., 2015, 2018). En el primero de estos trabajos, los autores analizaron las tablas estadísticas en cuatro libros de texto de $1^{\circ}$ y $2^{\circ}$ de educación primaria de dos editoriales chilenas. Entre las variables consideraron los niveles de lectura propuestos por Curcio y colaboradores (Curcio, 1989; Friel et al., 2001), observando la presencia de solo los dos primeros niveles de lectura, leer los datos $(44,8 \%)$ y leer entre los datos $(55,2 \%)$, mientras que el contexto mayormente utilizado, entre los propuestos en las pruebas PISA (OECD, 2019), fue el de tipo personal (69\%), seguido del público (19\%). En el análisis realizado a otros tres libros de texto dirigidos a $3^{\circ}$ curso de primaria (Díaz-Levicoy et al., 2018), se observó que el contexto personal apareció con más frecuencia (83,5\%), en este caso los niveles de lectura no fueron considerados. 
García-García et al. (2019) analizan doce libros de texto mexicanos de educación primaria $\left(1^{\circ}\right.$ a $\left.6^{\circ}\right)$ de dos editoriales diferentes, considerando entre las variables los niveles de lectura y el tipo de contexto en que se presenta la tabla. Aunque dicho estudio entrega resultados generales, se pudo apreciar la alta presencia de los dos primeros niveles de lectura (leer datos y leer dentro de los datos) en la mayoría de los textos, el nivel leer más allá de los datos estuvo ausente en las actividades propuestas, mientras que el nivel leer detrás de los datos solo estuvo presente de $2^{\circ}$ a $6^{\circ}$ curso en una de las editoriales analizadas. Respecto al contexto, de los considerados por PISA (OECD, 2019), apareció con mayor fuerza el de tipo personal.

Un antecedente inmediato (Pallauta et al., 2021) es el análisis realizado a las tareas escolares relacionadas con las tablas estadísticas presentadas en textos escolares chilenos de $5^{\circ}$ a $8^{\circ}$ básico (10 a 13 años) de dos editoriales que se distribuyen gratuitamente en el sistema público y subvencionado de Chile. El análisis de contenido de las tareas contempló, entre otras variables, el contexto, de los planteados en el informe PISA, y el nivel de lectura requerido para desarrollar cada actividad. Se observó una predominancia del nivel de lectura L2, leer dentro de los datos (77,4\%), seguido del nivel L1, leer los datos (19,4\%). En cuanto a los contextos, el que apareció con mayor fuerza fue el de tipo personal (41,7\%), seguido del contexto ocupacional o laboral (16,8\%); los autores añaden los experimentos aleatorios como nuevo contexto por su presencia significativa $(16,1 \%)$ en las actividades planteadas.

En este trabajo se analiza el nivel de lectura junto al contexto en que se presenta la tabla estadística en una nueva muestra de libros de texto chilenos y otra de textos españoles, los primeros dirigidos a $7^{\circ}$ y $8^{\circ}$ curso de enseñanza básica, y los segundos a $1^{\circ}$ y $2^{\circ}$ de educación secundaria (12 a 14 años), con el objeto de comparar los resultados de ambos países. Puesto que no tenemos suficientes antecedentes del tratamiento de las tablas estadísticas en estos niveles educativos y en textos españoles, este trabajo aporta información original.

\section{Metodología}

La muestra se compone de seis libros de texto chilenos dirigidos a $7^{\circ}$ y $8^{\circ}$ básico (10 y 11 años) y seis españoles de $1^{\circ}$ y $2^{\circ}$ de educación secundaria, tres por cada nivel escolar analizado. La muestra de textos seleccionada (detallada en el Apéndice) es intencional, y se ajusta a criterios enmarcados por el objetivo del presente estudio (Corral et al., 2015). La codificación empleada para referirse a cada texto al incluir los ejemplos mostrados en el trabajo denota el tipo de enseñanza de cada país. Así, por ejemplo, el código EGB (Educación General Básica) se refiere a los libros de texto chilenos y cuando se indica ESO (Educación Secundaria Obligatoria) corresponde a cada uno de los textos españoles.
La muestra de libros de texto chilenos forma parte de tres series diferentes: una subvencionada por el Ministerio de Educación en Chile, publicada por dos editoriales diferentes en edición especial, SM (70 básico) y Santillana ( $8^{\circ}$ básico), por otro lado, hay textos editados por SM y Santillana directamente. Estos textos son distribuidos de forma gratuita a los estudiantes del sistema público y concertado. Además, en la muestra se incluyen otros textos editados por Santillana y SM que siguen las recomendaciones oficiales (MINEDUC, 2015), los cuales se encuentran vigentes y disponibles en el mercado. El criterio de la selección de estos textos fue su uso generalizado en las escuelas concertadas y privadas, además de haber sido publicados por editoriales de tradición en el país. La muestra de libros de texto españoles ha sido publicada por las editoriales Anaya, Edelvives y Santillana, que son ampliamente utilizadas en las diferentes comunidades autónomas de España, y atienden al marco curricular vigente (MECD, 2015).

En los libros de texto se analizaron las actividades o ejemplos que requerían el uso o construcción de una tabla estadística. En la Tabla 1 se presenta la distribución de las situaciones analizadas por curso y país. En total, se revisó un número importante de situaciones $(n=1174)$, siendo similar la cantidad de actividades analizadas por cada país. En Chile es muy superior la cantidad de actividades incluidas en los textos analizados en $7^{\circ}$ curso $(69,2 \%)$ que en $8^{\circ}(30,8 \%)$, lo que puede deberse a que el estudio explícito de la tabla está propuesto en $7^{\circ}$ básico, mientras que en el siguiente curso es un medio para la enseñanza de otros conceptos. En España, al contrario, el mayor número de situaciones se encuentran en $2^{\circ}$ curso $(56,3 \%)$ que en $1^{\circ}$ curso de ESO $(43,7 \%)$.

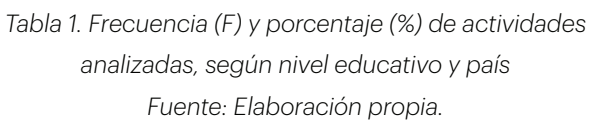

\begin{tabular}{|l|l|l|l|l|l|l|}
\hline \multirow{2}{*}{$\begin{array}{l}\text { Nivel } \\
\text { educativo }\end{array}$} & \multicolumn{2}{l|}{$\begin{array}{l}\text { Textos } \\
\text { chilenos }\end{array}$} & \multicolumn{2}{l|}{$\begin{array}{l}\text { Textos } \\
\text { españoles }\end{array}$} & \multicolumn{2}{l|}{ Total } \\
\cline { 2 - 8 } & F & $\%$ & F & $\%$ & F & $\%$ \\
\hline $7^{\circ} \mathrm{EB} / 1^{\circ} \mathrm{ESO}$ & 393 & 69,2 & 265 & 43,7 & 658 & 56 \\
\hline $\begin{array}{l}8^{\circ} \mathrm{EB} / 2^{\circ} \\
\mathrm{ESO}\end{array}$ & 175 & 30,8 & 341 & 56,3 & 516 & 44 \\
\hline Total & 568 & 100 & 606 & 100 & 1174 & 100 \\
\hline
\end{tabular}

Para el análisis de las situaciones problema presentadas en los libros de texto se utilizó un análisis de contenido (Díaz-Herrera, 2018), el que permite profundizar en las características de documentos escritos, en este caso el libro de texto. El primer paso consiste en la selección de las unidades de análisis: para ello se identifica, en el tema o unidad didáctica de estadística y probabilidad de cada texto que compone la muestra, los párrafos que contuvieran situaciones en donde se hiciera uso de la tabla estadística. 
Seguidamente, se determina la variable de análisis, en este caso cada una de las actividades, y en ellas se analiza el nivel de lectura y contexto. De este modo, mediante un proceso cíclico e inductivo (Bisquerra, 2014), se clasifican las actividades en que se involucra cualquier tipo de tabla estadística en las categorías definidas, seleccionando ejemplos de cada una de ellas. Se realizan continuas revisiones de los textos por parte de los autores, discutiendo los desacuerdos de codificación, para llegar a un consenso asegurando así la fiabilidad del análisis realizado. Finalmente, se elaboran tablas para resumir los resultados obtenidos y facilitar la obtención de conclusiones.

\section{Resultados}

En el siguiente apartado se presentan los resultados obtenidos en el análisis del nivel de lectura requerido junto al contexto en que es planteada la tabla estadística, en la muestra de libros de texto chilenos y españoles.

\subsection{Niveles de lectura}

En primer lugar, se describen los resultados sobre el nivel de lectura encontrados en los libros de texto analizados, utilizando los niveles de lectura propuestos por Friel et al. (2001). Se comienza describiendo con detalle las categorías de análisis, incorporando ejemplos que clarifiquen cada categoría, siguiendo con los resultados en los textos chilenos y luego en los textos españoles, para finalmente describir la comparación entre ambos países.

L1. Leer los datos. En este nivel, la respuesta a la pregunta planteada únicamente implica una lectura literal de la información que es explícita en la tabla, por lo que no se requiere realizar cálculos o ninguna otra operación con los datos representados.

El ejemplo reproducido en la Figura 1 presenta una actividad seleccionada de un libro de texto chileno dirigido a 70 básico, en el que luego de completar la tabla de distribución de una variable cualitativa con frecuencias ordinarias (absolutas, relativas y porcentuales), el estudiante debe responder a una serie de cuestiones entre ellas para identificar la variable de estudio y su tipo. Este tipo de preguntas es importante, pues de acuerdo a la variable representada se podría, posteriormente, determinar la pertinencia de calcular medidas de centralización como la media, moda o mediana. Otro tipo de pregunta que se plantea es identificar la frecuencia porcentual de una determinada categoría de la variable, y explicar cómo se obtuvo la respuesta, donde basta con indicar la columna utilizada para ello. Ambas cuestiones señaladas requieren de una lectura literal de la tabla, por lo que se enmarcarían en el nivel más básico de lectura, de acuerdo a Friel et al. (2001).

\begin{abstract}
Se introducen las diferentes piezas de un ajedrez en una caja y se extrae una al azar, se anota qué pieza es y se devuelve a la caja.

Este experimento aleatorio se ha repetido 40 veces y se han obtenido los siguientes resultados:

\begin{tabular}{|c|c|c|c|c|c|c|}
\hline & Peón & Torre & Caballo & Alfil & Rey & Reina \\
\hline $\begin{array}{c}\text { Frecuencia } \\
\text { absoluta, } n\end{array}$ & 7 & 5 & 10 & 3 & 8 & 7 \\
\hline
\end{tabular}

a. ¿Cuál es el espacio muestral del experimento?

b. ¿Cuál es la frecuencia absoluta de cada una de las piezas?
\end{abstract}

Figura 1. Ejemplo de actividad con nivel de lectura L1, leer los datos. Fuente: EGB5 (p. 221).

L2. Leer dentro de los datos. En este nivel, la actividad no solo implica la lectura literal, sino que también se precisa comparar datos representados en la tabla, o aplicar cálculos con ellos. Se requiere de este nivel, por ejemplo, para determinar las medidas de centralización y rango de una distribución. Un ejemplo se presenta en la Figura 2, que es una actividad resuelta recogida de un libro de texto español dirigido a $2^{\circ}$ ESO. En ella se explica al estudiante los pasos necesarios para calcular la media aritmética mostrando, además, la fórmula de cálculo y utilizando simbolización, e incluso subíndices. Para seguir el proceso de cálculo, el estudiante ha de leer en la tabla las modalidades de la variable y su frecuencia (absoluta y absoluta acumulada) correspondiente, que debe identificar en la tabla. Además, necesita comprender dichos conceptos para realizar correctamente las operaciones que se hacen con sus valores, según las columnas respectivas en la tabla, lo cual corresponde al nivel de leer dentro de los datos, ya que se pide al estudiante obtener información que no está representada literalmente en la tabla. Resaltamos el hecho de que se utiliza la notación simbólica (subíndices) para representar el valor de la variable xi, que se particulariza como x1, x2, x3 en la fórmula; de este modo, el estudiante debe deducir que i indica el orden de los valores de la variable en la tabla y a su vez informa del valor de la variable correspondiente a dicho subíndice en dicha fórmula. 
La variable Número de intentos es una variable cuantitativa.

$$
\begin{aligned}
& \begin{array}{|c|c|c|c|}
\hline x_{i} & f_{i} & F_{i} & f_{i} \cdot x_{j} \\
\hline 1 & 27 & 27 & 1 \cdot 27=27 \\
\hline 2 & 65 & 27+65=92 & 2 \cdot 65=130 \\
\hline 3 & 8 & 92+8=100 & 3 \cdot 8=24 \\
\hline \text { Total } & 27+65+8=100 & & 27+130+24=181 \\
\hline
\end{array} \\
& \text { - Media aritmética } \quad \bar{x}=\frac{f_{1} \cdot x_{1}+f_{2} \cdot x_{2}+f_{3} \cdot x_{3}}{N}=\frac{181}{100}=1,81 \\
& \text { - Mediana El valor central ocupará la posición: } \frac{N}{2}=50 \text {. Por las } \\
& \text { son 2. } \rightarrow M e=(2+2) / 2=2
\end{aligned}
$$

Figura 2. Ejemplo de actividad con nivel de lectura L2, leer dentro de los datos.

Fuente: ESO3 (p. 283)

L3. Leer más allá de los datos. Se trata de actividades que implican una mayor capacidad de lectura de la tabla estadística, pues se pide inferir información no representada y que no es posible extraer mediante operaciones aritméticas. Un ejemplo sería interpolar o extrapolar un valor en una serie de datos ordenados a lo largo del tiempo. En la Figura 3 se reproduce una actividad de un libro de texto chileno que incluye una pregunta cuya respuesta requiere este nivel de lectura. Se debe conjeturar, basándose en los datos de la tabla, el valor de la frecuencia relativa de cada modalidad de la variable si se incrementan las extracciones de una urna con fichas numeradas de 1 a 10. Para responder la pregunta, no solo se deben leer los valores de la frecuencia que ha correspondido a cada ficha numerada en la tabla (nivel de lectura 1), y comprobar que el valor de cada una es, aproximadamente, la décima parte de las extracciones (nivel de lectura 2). Además, se debe inferir que esta similitud de frecuencias se conservará en nuevos lanzamientos, por lo que, al realizar los 100.000 lanzamientos, aproximadamente cada número saldrá 10.000 veces. Además, hay que comprender que los resultados deben tener cierta variabilidad. Por tanto, se requiere un nivel de lectura 3 .

Completa la tabla en tu cuaderno considerando que se realizaron 5000 extracciones de una ficha desde una urna con fichas numeradas desde 5000 extracciones de una fi
el 1 al 10 . Luego, resuelve.

\begin{tabular}{|c|c|c|c|c|c|c|c|c|c|c|}
\hline \multicolumn{10}{|c|}{5000 extracciones } \\
\hline $\mathrm{N}^{\circ}$ & 1 & 2 & 3 & 4 & 5 & 6 & 7 & 8 & 9 & 10 \\
\hline$f$ & 490 & 513 & 501 & 491 & 508 & 506 & 493 & 498 & 502 & 498 \\
\hline$f_{r}$ & $\square$ & $\square$ & $\square$ & $\square$ & $\square$ & $\square$ & $\square$ & $\square$ & $\square$ & $\square$ \\
\hline
\end{tabular}

c. Si se aumentan las extracciones a 100000 , ¿a qué valor debiese tender cada probabilidad frecuencial? ¿Por qué?

Figura 3. Ejemplo de actividad con nivel de lectura L3, leer más allá de los datos. Fuente: EGB1 (p. 213).
L4. Leer detrás de los datos. Corresponde al nivel más avanzado, que implica no solo la lectura del gráfico o tabla, sino también ser capaz de realizar una valoración crítica de su contenido, de las fuentes de las que se ha extraído la información, o de la veracidad de las afirmaciones realizadas sobre su contenido (Shaughnessy et al., 1996). En la Figura 4 se reproduce un ejemplo de un libro de texto español, en el que se pide al estudiante tomar una decisión a partir de la información entregada en tres tablas de frecuencia. Para ello, se debe discutir si tres escolares ficticios han realizado la tarea descrita correctamente, aunque todos alegaran que lo han hecho. Para ello, los estudiantes deben relacionar la amplitud de los sectores de la ruleta dibujada en el enunciado, uno de los cuales ocupa la mitad del área y, en consecuencia, aproximadamente, el color rojo ha de obtenerse la mitad de las veces (180). De los otros dos sectores, uno ocupa dos sextos del área y el otro uno, por lo cual los valores aproximados de veces en que aparecen los colores azul y amarillo serán 120 y 60, de lo que se deduce que Adrián ha hecho trampa. Puesto que no es de esperar unos valores exactos, Carla también ha debido inventar sus datos. En consecuencia, además de leer las tablas y comparar los datos con los esperados al realizar el experimento, se debe observar de manera crítica los resultados presentados en cada muestra y argumentar la respuesta, lo que supone el nivel superior de lectura.
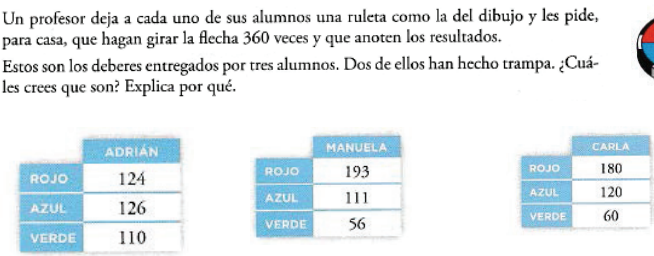

Figura 4. Ejemplo de actividad con nivel de lectura L4, leer

detrás de los datos.

Fuente: ESO1 (p. 303)

\subsubsection{Resultados en los libros de textos chilenos}

La distribución de los diferentes niveles de lectura presentados en las actividades propuestas en los libros de texto chilenos dirigidos a $7^{\circ}$ y $8^{\circ}$ básico se resume en la Tabla 2. Globalmente, el nivel de lectura que aparece con mayor frecuencia es $L 2$, leer entre los datos $(75,4 \%)$, que supone la realización de comparaciones y cálculo a partir de los datos de la tabla. Sigue el nivel L1, leer los datos (16,4\%), es decir, la lectura directa sin operaciones posteriores. Los niveles más sofisticados de lectura como L3, leer más allá de los datos $(2,6 \%)$ y L4, leer detrás de los datos $(5,6 \%)$, que requieren interpolación o extrapolación y lectura crítica de la tabla, aparecen en un menor porcentaje. 
Tabla 2. Frecuencia (F) y porcentaje (\%) de niveles de lectura por nivel educativo. Fuente: Elaboración propia

\begin{tabular}{|l|l|l|l|l|l|l|}
\hline \multirow{2}{*}{$\begin{array}{l}\text { Nivel de } \\
\text { lectura }\end{array}$} & \multicolumn{3}{|l|}{ Educación Básica } & \multicolumn{2}{l}{ Total } \\
\cline { 2 - 7 } & \multicolumn{2}{|l|}{$\mathbf{7 0}^{\prime}$ EB } & \multicolumn{2}{l|}{$\mathbf{8}^{\circ}$ EB } & \multicolumn{2}{l|}{} \\
\cline { 2 - 7 } & F & $\%$ & F & $\%$ & F & $\%$ \\
\hline $\begin{array}{l}\text { L1. Leer los } \\
\text { datos }\end{array}$ & 66 & 16,8 & 27 & 15,4 & 93 & 16,4 \\
\hline $\begin{array}{l}\text { L2. Leer } \\
\text { dentro de los } \\
\text { datos }\end{array}$ & 312 & 79,4 & 116 & 66,3 & 428 & 75,4 \\
\hline $\begin{array}{l}\text { L3. Leer más } \\
\text { allá de los } \\
\text { datos }\end{array}$ & 10 & 2,5 & 5 & 2,9 & 15 & 2,6 \\
\hline $\begin{array}{l}\text { L4. Leer } \\
\text { detrás de los } \\
\text { datos }\end{array}$ & 5 & 1,3 & 27 & 15,4 & 32 & 5,6 \\
\hline Total & 393 & 100 & 175 & 100 & 568 & 100 \\
\hline
\end{tabular}

Hay un ligero aumento de nivel al progresar el curso, pues en 70 básico el nivel que se presenta en mayor porcentaje es L2, leer entre los datos (79,4\%), mientras los niveles superiores de lectura como L3, leer más allá de los datos (2,5\%) y L4, leer detrás de los datos $(1,3 \%)$, se presentan en menor frecuencia. Aunque en $8^{\circ}$ básico también es más frecuente el nivel L2, leer entre los datos $(66,3 \%)$, su frecuencia disminuye con respecto al curso anterior, mientras que, en este nivel educativo, la lectura crítica de la tabla, es decir, el nivel L4, leer detrás de los datos $(15,4 \%)$, se presenta en un porcentaje mayor que en 70 básico, con igual porcentaje que el nivel L1, leer los datos.

En la Figura 5 se analizan los niveles de lectura en las tres editoriales revisadas en el trabajo. En la figura se aprecia que en las tres editoriales predominan las actividades de nivel de lectura $\mathrm{L} 2$, leer entre los datos (MINEDUC, 74,4\%; Santillana, 77,7\%; SM, 74,5\%), mientras que el nivel más básico de lectura L1, leer los datos, se presenta en un mayor porcentaje en los textos entregados por el MINEDUC (21,9\%), seguido de la editorial Santillana (14,5\%). Como se ha dicho, los niveles más avanzados de lectura son poco utilizados, siendo la editorial SM la que incluye un mayor porcentaje de actividades en los niveles L3, leer más allá de los datos $(5,1 \%)$ y $L 4$, leer detrás de los datos $(8,7 \%)$.

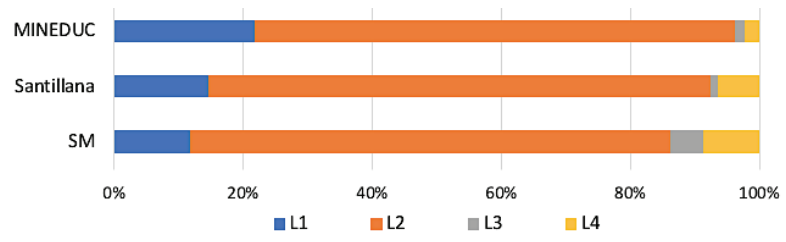

Figura 5. Porcentaje de actividades con diferentes niveles de lectura por editoriales chilenas.

Fuente: Elaboración propia.

\subsubsection{Resultados en los libros de texto españoles}

La Tabla 3 resume los resultados obtenidos en el análisis de los textos españoles respecto al nivel de lectura presentado en las actividades en los dos cursos analizados. Al igual como ocurrió en los textos chilenos, globalmente es más frecuente el segundo nivel, leer entre los datos (79\%), seguido por el nivel inicial L1, leer los datos (19,8\%). Los niveles superiores L3, leer más allá de los datos $(0,2 \%)$ y L4, leer detrás de los datos (1\%) son muy poco frecuentes.

Tabla 3. Frecuencia (F) y porcentaje (\%) de niveles de lectura por nivel educativo. Fuente: Elaboración propia.

\begin{tabular}{|c|c|c|c|c|c|c|}
\hline \multirow{3}{*}{$\begin{array}{l}\text { Nivel de } \\
\text { lectura }\end{array}$} & \multicolumn{4}{|c|}{ Educación Secundaria } & \multirow{2}{*}{\multicolumn{2}{|c|}{ Total }} \\
\hline & \multicolumn{2}{|c|}{$1^{\circ} \mathrm{ESO}$} & \multicolumn{2}{|c|}{$2^{\circ} \mathrm{ESO}$} & & \\
\hline & $\mathbf{F}$ & $\%$ & $\mathbf{F}$ & $\%$ & $\mathbf{F}$ & $\%$ \\
\hline $\begin{array}{l}\text { L1. Leer los } \\
\text { datos }\end{array}$ & 48 & 18,1 & 72 & 21,1 & 120 & 19,8 \\
\hline $\begin{array}{l}\text { L2. Leer } \\
\text { dentro de los } \\
\text { datos }\end{array}$ & 215 & 81,1 & 264 & 77,4 & 479 & 79,0 \\
\hline $\begin{array}{l}\text { L3. Leer más } \\
\text { allá de los } \\
\text { datos }\end{array}$ & 1 & 0,4 & & & 1 & 0,2 \\
\hline $\begin{array}{l}\text { L4. Leer } \\
\text { detrás de los } \\
\text { datos }\end{array}$ & 1 & 0,4 & 5 & 1,5 & 6 & 1 \\
\hline Total & 265 & 100 & 341 & 100 & 606 & 100 \\
\hline
\end{tabular}

Al comparar la distribución de niveles de lectura en ambos cursos, sigue siendo el segundo nivel L2, leer entre los datos, el que aparece con mayor fuerza, aunque disminuye ligeramente en el segundo curso (10 ESO, 81,1\%; $2^{\circ}$ ESO, 77,4\%). Le sigue el nivel inicial L1, leer los datos, que es algo más frecuente en $2^{\circ}$ curso $(21,1 \%)$ que en 10 ESO $(18,1 \%)$. El nivel L3, leer más allá de los datos, aparece de manera muy escasa en $1^{\circ}$ curso $(0,4 \%)$ y no se presenta en $2^{\circ}$ curso de ESO, mientras que L4, leer detrás de los datos, tiene una presencia algo más en $2^{\circ}$ curso $(1,5 \%)$ que en 10 curso ESO $(0,4 \%)$.

La Figura 6 muestra la distribución de los niveles de lectura en las tres editoriales españolas analizadas. Observamos que las tres colecciones priorizan en las actividades el nivel de lectura L2, leer entre los datos (Anaya, 84,9\%; Edelvives, 74,3\%; Santillana, 77,7\%), que prácticamente abarca la totalidad de las actividades, debido a que la lectura de tablas en los textos se usa como paso previo para el cálculo de estadísticos como la media, moda o el rango. En segundo lugar, de importancia se incluye el nivel L1, leer los datos; dicho nivel aparece menos en Anaya (14,6\%), en comparación a las demás editoriales. Los niveles de 
lectura superiores apenas aparecen: notamos que L3, leer más allá de los datos, es recogido únicamente y de manera muy limitada por Edelvives $(0,4 \%)$, esta última editorial es la que presenta un porcentaje ligeramente superior de actividades que implican el máximo nivel de lectura L4, leer detrás de los datos (2,2\%), dicho nivel también es recogido por Anaya, pero de forma muy escasa $(0,5 \%)$.

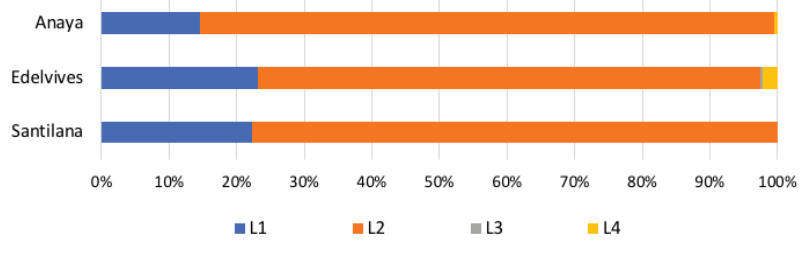

Figura 6. Porcentaje de niveles de lectura por nivel educativo y editoriales españolas. Fuente: Elaboración propia.

\subsubsection{Comparación de resultados en los dos países}

Para completar el análisis de las secciones anteriores, en la Figura 7 se presenta un gráfico comparativo con la distribución de los niveles de lectura por nivel educativo y país. Observamos que los libros de texto chilenos presentan una mayor proporción de actividades de niveles L3, leer más allá de los datos y L4, leer detrás de los datos que los españoles. También se aprecia un incremento de los niveles de lectura conforme se progresa de curso, pues en $7^{\circ}$ básico los niveles L3, leer más allá de los datos $(2,5 \%)$ y L4, leer detrás de los datos $(1,4 \%)$ aparecen de manera muy escasa, pero en $8^{\circ}$ se incrementan de manera significativa (L3, 2,9\%; L4, 15,4\%).

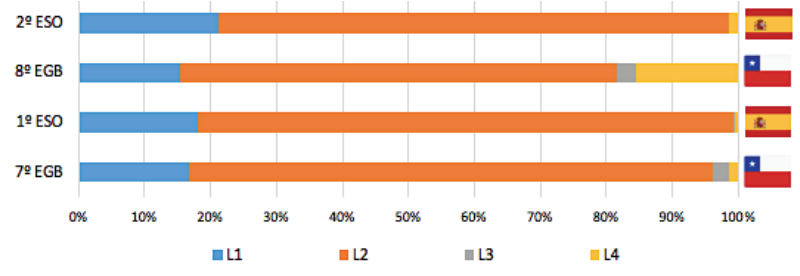

Figura 7. Porcentaje de actividades con diferentes niveles de lectura por nivel educativo y país. Fuente: Elaboración propia.

Los libros de texto españoles también muestran un incremento del nivel máximo de lectura $L 4$, leer detrás de los datos (10 ESO, 0,4\%; $2^{\circ} \mathrm{ESO}, 1,5 \%$ ), aunque de manera más ligera, conforme se avanza de nivel educativo; sin embargo, L3, leer más allá de los datos no es contemplado en $2^{\circ}$ curso. En estos textos se prioriza actividades que requieren de los niveles de lectura literal, por ejemplo, L1, leer los datos se incrementa de $1^{\circ}$ curso $(18,1 \%)$ a $2^{\circ}$ curso ESO $(21,1 \%)$, mientras que $\mathrm{L} 2$, leer entre los datos disminuye ligeramente (10 ESO, $\left.81,1 \% ; 2^{\circ} \mathrm{ESO}, 77,4 \%\right)$.
En resumen, los resultados obtenidos del análisis muestran una predominancia de actividades que requieren los niveles más básicos de lectura, L1, leer los datos y L2, leer entre los datos en los dos países, que coinciden con los resultados obtenidos por DíazLevicoy et al. $(2015,2018)$ con libros de texto de los primeros años de educación primaria en Chile. En nuestro caso es mayor la proporción del nivel L2, leer entre los datos, y menor del nivel L1, leer los datos respecto a los datos obtenidos por dichos autores, lo que se comprende al tratarse de cursos superiores a los que ellos analizaron. Lo mismo ocurrió en la investigación de García-García et al. (2019) en México, y en todas estas investigaciones los niveles L3, leer más allá de los datos y L4, leer detrás de los datos no se consideraron, también razonable porque dichos trabajos se dirigen a niveles educativos inferiores a los tratados en, por ejemplo, nuestro caso. En nuestro análisis anterior de otros textos chilenos (Pallauta et al., 2021) se obtuvieron resultados muy similares en cuanto a la alta presencia de los niveles más básicos de lectura, mientras en el presente estudio se observa un incremento de los niveles más sofisticados de lectura, especialmente en $8^{\circ}$ básico.

\subsection{Contextos}

Seguidamente se presentan los resultados del análisis de los contextos en que se plantean los problemas en los textos de la muestra. Comenzamos describiendo las categorías de contextos, que corresponden a los planteados en los estudios PISA (OECD, 2019), a las que se ha añadido "experimento aleatorio" como un nuevo contexto, debido a su uso en los textos analizados para el estudio de la probabilidad frecuencial. Luego se presenta la distribución de contextos en los textos chilenos y españoles, para finalizar con una comparación entre ambas colecciones de textos.

Contexto personal. Entre las situaciones consideradas en las pruebas PISA, las de tipo personal se refieren a las cercanas a la experiencia inmediata del estudiante (Turner, 2006). Son las actividades propias del cotidiano, o las que puede observar en su entorno inmediato. Por ejemplo, en la Figura 8 se presenta la distribución de los deportes favoritos de un grupo de estudiantes. Otros contextos personales encontrados en los textos son las actividades que prefieren los estudiantes realizar en su tiempo libre, o la cantidad de goles anotados por su equipo favorito en un partido de fútbol.

El primer día de clase tras las vacaciones, Celia y sus compañeros han rellenado una ficha con sus datos personales, entre ellos la edad de cada uno. Las edades recogidas han sido las siguientes:

$\begin{array}{llllllllllll}14 & 14 & 15 & 13 & 15 & 14 & 14 & 14 & 14 & 15 & 13 & 14\end{array}$ $\begin{array}{lllllllllllll}15 & 16 & 14 & 15 & 13 & 14 & 15 & 13 & 14 & 14 & 14 & 15 & 14\end{array}$

Elabora una tabla de frecuencias.

Figura 8. Ejemplo de actividad de contexto personal.

Fuente: ESO5 (p. 200). 
Contexto laboral. Son situaciones algo más apartadas de lo personal, que cualquiera podría encontrar en una actividad diaria organizada, como el trabajo (Turner, 2006). Es decir, las actividades propias del quehacer ocupacional o profesional que se desarrollan en diversos ámbitos. La Figura 9 es una actividad seleccionada de un libro de texto español, dirigida a $2^{\circ}$ curso de secundaria, y muestra la cantidad de libros vendidos por los tres dependientes de una librería. A partir de esta información se pide determinar algunos resúmenes estadísticos.

En una librería, el número de libros que han vendido tres trabajadores en una semana de trabajo ha sido el siguiente:

\begin{tabular}{|l|l|l|l|l|l|l|}
\hline & L & M & X & J & V & S \\
\hline Anna & 4 & 2 & 7 & 3 & 8 & 3 \\
\hline Quim & 5 & 4 & 6 & 6 & 4 & 3 \\
\hline Clara & 4 & 4 & 4 & 4 & 4 & 4 \\
\hline
\end{tabular}

a) Calcula las medidas estadisticas de lo que ha vendido cada trabajador.

Figura 9. Ejemplo de actividad de contexto ocupacional o laboral. Fuente: ESO6 (p. 291)

Contexto público. Se trata de las situaciones presentes en la comunidad y el entorno del estudiante, que se pueden observar en el entorno local o en contextos más amplios, a las que es posible de acceder a través de los diferentes medios de comunicación. En la Figura 10 se muestra un ejemplo recogido de un libro de texto chileno de $7^{\circ}$ básico en que se exponen los resultados sobre una consulta, realizada por un organismo gubernamental, en que se les pide a los jóvenes caracterizar a las personas que viven en situación de pobreza. Posteriormente, con dicha información, el estudiante debe construir una tabla de distribución.

El $41 \%$ de los jóvenes opinan que la mayoría de las personas en situación de pobreza en Chile son personas que no alcanzan condiciones de vida dignas en salud, trabajo, educación y vivienda. El $23 \%$ de los entrevistados opina que son personas que viven en hogares que no consiguen obtener un mínimo de ingreso al mes, mientras que el $17 \%$ opina que son personas que son discriminadas o excluidas por su origen social, cultura, género u otras razones. Es interesante observar que otro $17 \%$ de los entrevistados opina que son personas que no trabajan o no se esfuerzan. Un $2 \%$ no sabe o no responde.

Información extraída de: http://www.injuv.gob.cl/storage/docs/ Sondeo_Percepcion_de_los_jovenes_sobre_la_pobreza.pdf

Si los casos fueron 1010, construye una tabla de frecuencias que represente los datos mencionados.

Figura 10. Ejemplo de actividad de contexto público.

\section{Fuente: EGB1 (p. 194).}

Contexto científico. Incluyen aplicaciones de las matemáticas más abstractas y complejas que los anteriores contextos, y se asocian a procesos científicos o tecnológicos. Algunos ejemplos serían la duración de la inmunización tras recibir alguna de las dosis de la vacuna para el COVID-19, o la relación del grupo sanguíneo y el factor $\mathrm{RH}$ de un grupo de personas. En la Figura 11 se muestra un ejemplo de un libro de texto chileno dirigido a $8^{\circ}$ básico en que se presenta una tabla de datos con el registro de las temperaturas alcanzadas en el océano Pacífico según la latitud.

\begin{tabular}{|c|c|c|}
\hline \multirow{7}{*}{$\begin{array}{l}\text { Los rayos del sol caen de forma } \\
\text { más directa en las regiones } \\
\text { cercanas a la linea del ecuador, } \\
\text { durante casi todo el año. }\end{array}$} & \multicolumn{2}{|c|}{$\begin{array}{l}\text { La tabla muestra el promedio } \\
\text { de las temperaturas registradas } \\
\text { en el océano Pacífico según } \\
\text { sea la latitud. }\end{array}$} \\
\hline & \multicolumn{2}{|c|}{ Promedios de temperaturas } \\
\hline & Latitud & Temperatura $\left({ }^{\circ} \mathrm{C}\right)$ \\
\hline & $0^{\circ}, 10^{\circ}$ & 26,0 \\
\hline & $20^{\circ}, 30^{\circ}$ & 21,5 \\
\hline & $50^{\circ}, 60^{\circ}$ & 5,0 \\
\hline & $60^{\circ}, 70^{\circ}$ & $-1,3$ \\
\hline
\end{tabular}

Figura 11. Ejemplo de actividad de contexto científico. Fuente: EGB6 (p. 266).

Experimento aleatorio. Al igual que Pallauta et al. (2021), incorporamos esta categoría al análisis del contexto debido a su alta presencia en actividades en que se presentan los resultados en juegos de azar o experiencias aleatorias, que son planteadas para el estudio de la probabilidad, principalmente, desde su enfoque frecuencial (Ortiz, 2002). Las Figuras 1, 3 y 4 son ejemplos de ello.

Sin contexto. Consideramos en esta última categoría las actividades en que no se presenta el contexto en que fueron extraídos los datos presentados en la tabla estadística, como en la Figura 12, que muestra un ejemplo de un libro de texto español de $2^{\circ}$ ESO. En este sentido, como señala Van den Heuvel-Panhuizen (2005), se debe tener en cuenta que una enseñanza desvinculada de las experiencias de los estudiantes podría significar que olviden de forma rápida lo aprendido, mientras que un aprendizaje producido mediante tareas en contexto promueve mejores opciones de comprensión de conceptos a largo plazo.

2. Copia esta tabla de frecuencias y complétala.
\begin{tabular}{|c|c|c|} 
Datos & Frecuencia absoluta & Frecuencia relativa \\
\hline 2 & & 0,25 \\
\hline 3 & 7 & \\
\hline 7 & & 0,125 \\
\hline 20 & 13 & \\
\hline & $N=32$ & \\
\hline
\end{tabular}

Figura 12. Ejemplo de actividad sin contexto. Fuente: ESO6 (p. 293) 


\subsubsection{Análisis de contextos en los textos chilenos}

Los diferentes tipos de contextos de las actividades planteadas respecto a las tablas estadísticas en los libros de texto chilenos son resumidos en la Tabla 4. Apreciamos que el contexto que aparece con mayor fuerza es el de tipo personal $(46,7 \%)$, que muestra el interés de los autores de los textos por acercar la estadística a los intereses inmediatos de los estudiantes. Sigue en frecuencia el contexto de experimento aleatorio (19,9\%) requerido en el trabajo con la probabilidad, y a continuación el público (15,1\%), relacionado con la comunidad del estudiante.

Tabla 4. Frecuencia (F) y porcentaje (\%) de tipo de contexto por nivel educativo. Fuente: Elaboración propia.

\begin{tabular}{|c|c|c|c|c|c|c|}
\hline \multirow[t]{3}{*}{ Contexto } & \multicolumn{4}{|c|}{ Educación Básica } & \multirow{2}{*}{\multicolumn{2}{|c|}{ Total }} \\
\hline & \multicolumn{2}{|c|}{$7^{\circ} \mathrm{EB}$} & \multicolumn{2}{|c|}{$8^{\circ} \mathrm{EB}$} & & \\
\hline & $\mathbf{F}$ & $\%$ & $\mathbf{F}$ & $\%$ & $\mathbf{F}$ & $\%$ \\
\hline Personal & 185 & 47,1 & 80 & 45,7 & 265 & 46,7 \\
\hline $\begin{array}{l}\text { Laboral/ } \\
\text { educativo }\end{array}$ & 19 & 4,8 & 31 & 17,7 & 50 & 8,8 \\
\hline Público & 67 & 17 & 19 & 10,9 & 86 & 15,1 \\
\hline Científico & 16 & 4,1 & 22 & 12,6 & 38 & 6,7 \\
\hline $\begin{array}{l}\text { Experimento } \\
\text { aleatorio }\end{array}$ & 93 & 23,7 & 20 & 11,4 & 113 & 19,9 \\
\hline Sin contexto & 13 & 3,3 & 3 & 1,7 & 16 & 2,8 \\
\hline Total & 393 & 100 & 175 & 100 & 568 & 100 \\
\hline
\end{tabular}

Al comparar el contexto en los dos cursos analizados, se puede apreciar que en $7^{\circ}$ básico se presenta más el contexto personal $(47,1 \%)$ que en $8^{\circ}$ curso $(45,7 \%)$, igual que ocurre con el experimento aleatorio ( $7^{\circ}$ curso, 23,7\%; $8^{\circ}$ básico, 11,4\%). En $8^{\circ}$ curso observamos que el contexto ocupacional aparece de manera significativa $(17,7 \%)$, a diferencia de 70 básico $(4,8 \%)$ y lo mismo ocurre con el contexto científico $(12,6 \%$ en $8^{\circ}$ frente a $4,1 \%$ en $7^{\circ}$ ). Pensamos que los autores de los textos han querido incidir más en estos temas más abstractos con los estudiantes de mayor edad. Las actividades sin contexto son poco utilizadas tanto en $7^{\circ}(3,3 \%)$ como en $8^{\circ}$ curso $(1,7 \%)$.

Los diferentes tipos de contextos, según editorial, son presentados en la Figura 13, en la que se puede notar que las editoriales priorizan el uso del contexto personal para plantear las tablas, siendo más frecuente en Santillana (52,2\%). El contexto laboral se presenta de manera similar en Santillana $(13,4 \%)$ y SM $(11,7 \%)$, mientras que MINEDUC lo recoge en menor medida $(2,8 \%)$, y en cambio le da mayor relevancia al contexto público, a diferencia de las demás editoriales. La categoría experimento aleatorio aparece de manera significativa en MINEDUC (24,2\%) y SM (25,5\%), y en Santillana tiene una menor frecuencia (7\%). El contexto científico no es incorporado por MINEDUC, a diferencia de Santillana que lo incluye en una proporción apreciable (18,5\%). Destacamos que las actividades sin contexto son muy escasas en MINEDUC $(0,9 \%)$ y Santillana $(0,6 \%)$, y se presentan algo más en $\operatorname{SM}(6,6 \%)$.

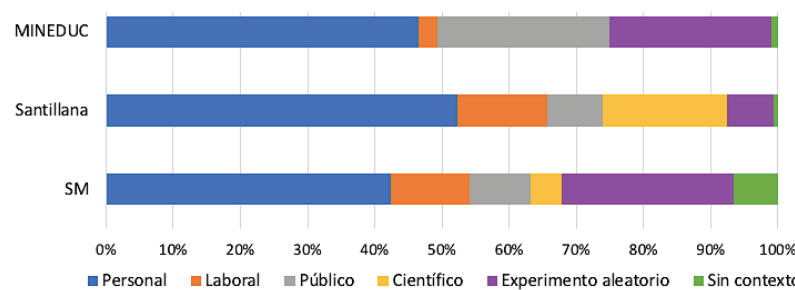

Figura 13. Porcentaje de contextos, según editorial chilena. Fuente: Elaboración propia.

\subsubsection{Análisis de contextos en los textos españoles}

La Tabla 5 resume los tipos de contextos encontrados en los libros de texto españoles. Al igual que en los textos chilenos, el contexto con mayor presencia es el personal $(48,8 \%)$. En el caso de los textos españoles el segundo más frecuente es el contexto público $(15,7 \%)$, seguido por el experimento aleatorio $(11,4 \%)$.

Al comparar los dos cursos de la muestra de textos, el contexto personal aparece con mayor fuerza en 10 curso $(57,7 \%)$ que en $2^{\circ}$ curso de la ESO $(41,9 \%)$; lo mismo ocurre con las actividades sin contexto que son más frecuentes en $1^{\circ}(11,3 \%)$ que en $2^{\circ}$ curso $(8,8 \%)$, lo cual va en contra de los principios didácticos, puesto que dichas actividades son más abstractas. El contexto experimento aleatorio también en estos textos tiene una presencia significativa, siendo más utilizado en $2^{\circ}$ curso $(12,6 \%)$ que en 10 curso de la ESO $(9,8 \%)$.

Tabla 5. Frecuencia (F) y porcentaje (\%) de tipo de contexto por nivel educativo. Fuente: Elaboración propia

\begin{tabular}{|l|l|l|l|l|l|l|}
\hline \multirow{2}{*}{ Contexto } & \multicolumn{3}{|l|}{ Educación Secundaria } & \multicolumn{2}{l}{ Total } \\
\cline { 2 - 7 } & \multicolumn{2}{|l}{ 10 ESO } & \multicolumn{2}{l}{$\mathbf{2}^{\circ}$ ESO } & \multicolumn{2}{l|}{} \\
\cline { 2 - 7 } & F & $\%$ & F & $\%$ & F & $\%$ \\
\hline Personal & 153 & 57,7 & 143 & 41,9 & 296 & 48,8 \\
\hline $\begin{array}{l}\text { Laboral/ } \\
\text { educativo }\end{array}$ & 11 & 4,2 & 41 & 12 & 52 & 8,6 \\
\hline Público & 38 & 14,3 & 57 & 16,7 & 95 & 15,7 \\
\hline Científico & 7 & 2,6 & 27 & 7,9 & 34 & 5,6 \\
\hline $\begin{array}{l}\text { Experimento } \\
\text { aleatorio }\end{array}$ & 26 & 9,8 & 43 & 12,6 & 69 & 11,4 \\
\hline Sin contexto & 30 & 11,3 & 30 & 8,8 & 60 & 9,9 \\
\hline Total & 265 & 100 & 341 & 100 & 606 & 100 \\
\hline
\end{tabular}


Al analizar la presencia de los diferentes tipos de contextos según editorial en los textos españoles, observamos en la Figura 14 que, al igual que las editoriales chilenas, se prioriza el uso del contexto personal, siendo Edelvives la que menos lo utiliza (37\%) respecto a las demás. El contexto laboral se presenta de manera similar en Santillana $(14,6 \%)$ y Edelvives (11,3\%), pero es muy escaso en Anaya (1,4\%). Por otra parte, experimento aleatorio aparece en todas las editoriales, pero con diferente peso, siendo más frecuente en Edelvives (20,4\%), seguido de Anaya $(8,7 \%)$ y con una escasa presencia en Santillana (1,9\%). Las actividades sin contexto son pocas en Edelvives (3\%), mientras que en Anaya (14,6\%) y Santillana $(13,4 \%)$ se presentan en un mayor porcentaje.

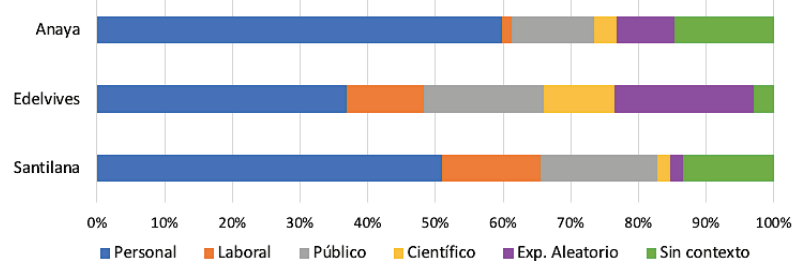

Figura 14. Porcentaje de contextos, según editorial española. Fuente: Elaboración propia.

\subsubsection{Comparación de resultados en los dos países}

En la Figura 15 se presenta un gráfico comparativo que muestra la distribución de los tipos de contextos, según nivel educativo y país. Apreciamos que en ambos países se prioriza el uso del contexto de tipo personal, lo que se enmarca en los lineamientos curriculares (MECD, 2015; MINEDUC, 2015), en cuanto a la importancia de proponer situaciones que sean cercanas para el estudiante. Este resultado coincide con otros análisis del contexto en que se proponen las tablas estadísticas en los libros de texto dirigidos a diferentes niveles educativos (Díaz-Levicoy et al., 2015, 2018; García-García et al., 2019; Pallauta et al., 2021).

En los textos chilenos se aprecia un incremento del contexto laboral conforme se progresa de curso y, de manera contraria, se disminuye la presencia de las actividades sin contexto al avanzar de $7^{\circ}$ a $8^{\circ}$ básico. En los libros de texto españoles se aprecia un incremento de los contextos laboral, científico y experimento aleatorio conforme se avanza de $1^{0}$ a $2^{\circ}$ ESO. Notamos que las actividades sin contexto tienen una mayor presencia en los libros españoles, a diferencia de los chilenos en que son menos utilizadas.

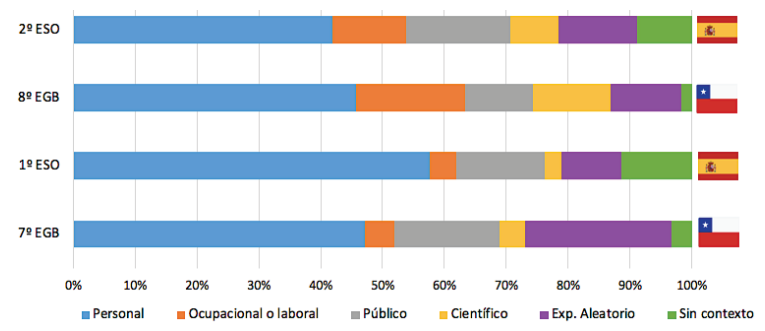

Figura 15. Porcentaje de contextos por nivel educativo y país.

Fuente: Elaboración propia.

\section{Conclusiones}

El análisis presentado proporciona nueva información referente al nivel de lectura requerido y el contexto en que se presentan las actividades sobre tablas estadísticas, en una muestra de libros de texto chilenos y españoles, siendo el primer estudio que se realiza sobre este tema en textos españoles, y complementando otro anterior (Pallauta et al., 2021) llevado a cabo con textos chilenos. Ambas variables han de ser tenidas en cuenta en la enseñanza, pues los niveles de lectura, de los propuestos por Curcio y colaboradores (Curcio, 1989; Friel et al., 2001), permiten identificar la demanda cognitiva requerida de las tareas propuestas, mientras que el contexto posee un rol relevante en el desarrollo del razonamiento estadístico de los estudiantes (Makar y Ben-Zvi, 2011).

Aunque los resultados sobre los niveles de lectura muestran un ligero incremento de los niveles de lectura más sofisticados, especialmente leer detrás de los datos, conforme se progresa de curso, especialmente en la muestra de textos chilenos, Ilama la atención la gran cantidad de actividades que se centran en los niveles más básicos de lectura. Aunque estos resultados coinciden con otros estudios, estos abordan niveles educativos inferiores (Díaz-Levicoy et al., 2015; García-García et al., 2019). Será importante entonces que, conforme se avanza de curso, se incremente el nivel de dificultad de las tareas, sobre todo fomentando una lectura crítica de los datos, necesaria como parte de la cultura estadística para tomar mejores decisiones, de cara al escenario de incertidumbre que enfrenta la sociedad en su conjunto (Rodríguez-Muñiz et al., 2020).

En relación a los contextos PISA (OECD, 2019), destacamos la alta presencia del contexto de tipo personal, tanto en los textos chilenos como españoles, que también concuerda con estudios anteriores (DíazLevicoy et al., 2015, 2018; García-García et al., 2019; Pallauta et al., 2021), y es sugerido en los lineamientos curriculares de ambos países (MECD, 2015; MINEDUC, 2015) en que se destaca la importancia de plantear contextos cercanos para el estudiante. Resaltamos también la frecuencia de uso del contexto experimento aleatorio, el cual fue incluido, al igual que en Pallauta et al. (2021), en el estudio de la probabilidad, pues 
las experiencias de juegos de azar forman parte del entorno cercano, y pueden permitir al profesor diseñar una enseñanza de la estadística basada en datos reales (Alsina y Annexa, 2021) que pueda ser más significativa para el estudiante. En este sentido, llama la atención la presencia de actividades que carecen de contexto, que, a pesar de ser escasas, especialmente en los textos chilenos, alerta de una tendencia que se pudiera incrementar en los cursos superiores, pues este tipo de actividades no facilita el equilibrio que debiera existir entre el contexto de una situación y la estadística que se aplica para resolverla (Wild y Pfannkuch, 1999).

La información presentada puede ser de interés para profesores, quienes la deben tener en cuenta en el diseño de la enseñanza de este tema, en particular. Puesto que la investigación sobre temas de estadística en educación secundaria, en general, es escasa, también se aporta información para la investigación en educación estadística, al realizar un comparativo de dos países sobre el tratamiento de la tabla estadística en los libros de texto. Como señalamos anteriormente, el libro de texto es ampliamente utilizado en el aula (Herbel, 2007), y en la actualidad toma mayor relevancia, pues en muchos casos es el único recurso con que cuentan los estudiantes, debido a que, en diferentes regiones y países, los procesos de instrucción no se están pudiendo realizar de manera presencial producto de la pandemia generada por el COVID-19.

\section{Agradecimientos}

Proyecto PID2019-105601GB-I00 / AEI / 10.13039/501100011033, Grupo FQM126 (Junta de Andalucía) y Beca ANID Folio: 72190280. 


\section{Referencias}

Alsina, Á., y Annexa, E. (2021). Estadística en contexto: Desarrollando un enfoque escolar común para promover la alfabetización. TANGRAM, 4(1), 71-98. https://doi.org/10.30612/tangram.v4i1.14396

Bisquerra, R. (2014). Metodología de la investigación educativa (6.a ed.). La Muralla.

Blum, W., Galbraith, P., Henn, H.-W., y Niss, M. (Eds.). (2007). Modelling and applications in mathematics education. Springer. https://doi.org/10.1007/978-0387-29822-1

Corral, Y., Corral, I., y Franco Corral, A. (2015). Procedimientos de muestreo. Revista Ciencias de la Educación, 26(46), 10-39.

Curcio, F. R. (1989). Developing graph comprehension. Elementary and middle school activities. National Council of Teachers of Mathematics.

Díaz-Herrera, C. (2018). Investigación cualitativa y análisis de contenido temático. Orientación intelectual de revista Universum. Revista General de Información y Documentación, 28(1), 119-142. https://doi.org/10.5209/ RGID.60813

Díaz-Levicoy, D., Morales, R., y López Martín, M. (2015). Tablas estadísticas en libros de texto chilenos de 10 y 20 año de educación primaria. Revista Paranaense de Educação Matemática, 4, 10-39. https://doi. org/10.30612/tangram.v1i2.7574

Díaz-Levicoy, D., Vásquez, C., y Molina-Portillo, E. (2018). Estudio exploratorio sobre tablas estadísticas en libros de texto de tercer año de educación primaria. Tangram, 1(2), 18-39. https://doi.org/10.30612/tangram. v1i2.7574

Estrella, S., y Estrella, P. (2020). Representaciones de datos en estadística: De listas a tablas. Revista Chilena de Educación Matemática, 12(1), 21-34. https://doi. org/10.46219/rechiem.v12i1.20

Font, V. (2007). Comprensión y contexto: Una mirada desde la didáctica de las matemáticas. Gaceta de la Real Sociedad Matemática Española, 10(2), 427-442.

Friel, S. N., Curcio, F. R., y Bright, G. W. (2001). Making sense of graphs: Critical factors influencing comprehension and instructional implications. Journal for Research in Mathematics Education, 32(2), 124-158. https://doi.org/10.2307/749671

Gal, I. (2019). Understanding statistical literacy: About knowledge of contexts and models. En J. M. Contreras, M. M. Gea, M. M. López-Martín, y E. Molina-Portillo (Eds.), Actas del Tercer Congreso Internacional Virtual de Educación Estadística. https://www.ugr.es/ fqm126/ civeest/ponencias/gal.pdf
García-García, J., Díaz-Levicoy, D., Vidal-Henry, S., y Arredondo, E. H. (2019). Las tablas estadísticas en libros de texto de educación primaria en México. Paradigma, 153-175. https://doi.org/10.37618/ PARADIGMA.1011-2251.2019.p153-175.id754

Gould, R. (2017). Data literacy is statistical literacy. Statistics Education Research Journal, 16(1), 22-25. https://doi.org/10.52041/serj.v16i1.209

Herbel, B. A. (2007). From Intended Curriculum to Written Curriculum: Examining the "Voice" of a Mathematics Textbook. Journal for Research in Mathematics Education, 38(4), 344-369. https://doi. org/10.2307/30034878

Makar, K., y Ben-Zvi, D. (2011). The role of context in developing reasoning about informal statistical inference. Mathematical Thinking and Learning, 13(1-2), 1-4. https://doi.org/10.1080/10986065.2011.538291

Ministerio de Educación, Cultura y Deporte de España. (2014). Real Decreto 126/2014, de 28 de febrero, por el que se establece el currículo básico de la Educación Primaria. Ministerio de Educación, Cultura y Deporte, Boletín Oficial del Estado Núm. 52.

Ministerio de Educación, Cultura y Deporte de España. (2015). Real Decreto 1105/2014, de 26 de diciembre, por el que se establece el currículo básico de la Educación Secundaria Obligatoria y del Bachillerato. Ministerio de Educación, Cultura y Deporte, Boletín Oficial del Estado Núm. 3.

Ministerio de Educación de Chile. (2015). Bases curriculares 70 básico a $2^{\circ}$ medio. Unidad de Currículum y Evaluación.

Ministerio de Educación de Chile. (2018). Bases curriculares Primero a Sexto Básico. Unidad de Currículum y Evaluación.

Organización para la Cooperación y el Desarrollo Económicos. (2019). PISA 2018 Mathematics Framework. En PISA 2018 Assessment and Analytical Framework (pp. 73-95). OECD. https://doi. org/10.1787/13c8a22c-en

Ortiz, J. J. (2002). La probabilidad en los libros de texto. Grupo FQM126.

Pallauta, J., y Arteaga, P. (2021). Niveles de complejidad semiótica en gráficos y tablas estadísticas. Números, $106,13-22$

Pallauta, J., Gea, M. M., y Arteaga, P. (2021). Caracterización de las tareas propuestas sobre tablas estadísticas en libros de texto chilenos de educación básica. Paradigma, 42, 32-60. https://doi.org/10.37618/ PARADIGMA.1011-2251.2021.p32-60.id1017 
Pepin, B., y Gueudet, G. (2020). Curriculum resources and textbooks in mathematics education. En Encyclopedia of mathematics education (pp. 87-94). Springer. $\quad$ https://doi.org/10.1007/978-3-030-157890_40

Reys, B., Reys, R., y Chavez, O. (2004). Why mathematics textbooks matter. Educational Leadership, 61(5), 61-66.

Rodríguez-Muñiz, L. J., Muñiz-Rodríguez, L., Vásquez, C., y Alsina, Á. (2020). ¿Cómo promover la alfabetización estadística y de datos en contexto? Estrategias y recursos a partir de la COVID-19 para Educación Secundaria. Números, 104, 217-238.

Rosales, E. M., Rodríguez, P. G., y Romero, M. (2020). Conocimiento, demanda cognitiva y contextos en la evaluación de la alfabetización científica en PISA. Eureka, 17(2), 2302. https://doi.org/10.25267/Rev_ Eureka_ensen_divulg_cienc.2020.v17.i2.2302.

Sanmartí, N., y Márquez, C. (2017). Aprendizaje de las ciencias basado en proyectos: Del contexto a la acción. Ápice. 1(1), 3-16. https://doi.org/10.17979/ arec.2017.1.1.2020.

Shaughnessy, J. M., Garfield, J., y Greer, B. (1996). Data handling. En A. Bishop, K. Clements, C. Keitel, J. Kilpatrick, y C. Laborde (Eds.), International handbook of mathematics education (Vol. 4, pp. 205-237). Springer. https://doi.org/10.1007/978-94-009-1465-0_8

Turner, R. (2006). El Programa Internacional para la Evaluación de los Alumnos (PISA). Una perspectiva general. Revista de Educación, nº extraordinario, 45-74.

Van den Heuvel-Panhuizen, M. (2005). The role of contexts in assessment problems in mathematics. For the Learning of Mathematics, 25(2), 2-23.

Wijaya, A., Van den Heuvel-Panhuizen, M., Doorman, M., y Robitzsch, A. (2014). Difficulties in solving context-based PISA mathematics tasks: An analysis of students' errors. The Mathematics Enthusiast, 11(3), $555-584$

Wild, C. J., y Pfannkuch, M. (1999). Statistical Thinking in Empirical Enquiry. International Statistical Review, 67(3), 223-248. https://doi.org/10.1111/j.1751-5823.1999. tb00442.x 


\section{Apéndice: Muestra de libros utilizados}

\section{Textos chilenos}

\begin{tabular}{|l|l|}
\hline Código & Referencia \\
\hline EGB1 & $\begin{array}{l}\text { Iturra, F., Manosalva, C., Ramírez, M., y Romero, D. (2019). 70 básico Matemática Texto del estudiante. SM. Edición } \\
\text { especial para el Ministerio de Educación. }\end{array}$ \\
\hline EGB2 & Marambio, V., y Castro, C. (2016). Matemática 70 básico, Proyecto Todos Juntos. Santillana. \\
\hline EGB3 & Schwerter, S., Aguilar, M., y Maulén, M. (2014). Sé protagonista, Matemática 70. SM. \\
\hline EGB4 & $\begin{array}{l}\text { Torres, C. y Caroca, V. (2019). 80 Básico Matemática Texto del estudiante. Santillana. Edición especial para el } \\
\text { Ministerio de Educación. }\end{array}$ \\
\hline EGB5 & Castro, C., Curiche, A., y Vega, M. (2014). Sé protagonista, Matemática 8. SM. \\
\hline EGB6 & Ramírez, A., Maldonado, L., Castro, C., y Ávila, J. (2016). Matemática 80 básico. Santillana. \\
\hline
\end{tabular}

\section{Textos españoles}

\begin{tabular}{|l|l|}
\hline Código & Referencia \\
\hline ESO1 & Colera, J., Gaztelu, I., y Colera R. (2016). ESO 1 Matemáticas (Proyecto Aprender es crecer en conexión). Anaya. \\
\hline ESO2 & Mejía D., Romero, R., y Ocaña, J. (2015). ESO 1 Matemáticas (Proyecto somoslink). Edelvives. \\
\hline ESO3 & $\begin{array}{l}\text { Almodóvar, J., De la Prida, C., Gaztelu, A., González, A., Machín, P., Pérez, C., y Sánchez, D. (2016). Matemáticas } \\
\text { Serie Resuelve ESO 1 (Proyecto Saber Hacer). Santillana. }\end{array}$ \\
\hline ESO4 & Colera, J., Gaztelu, I., y Colera R. (2017). ESO 2 Matemáticas (Proyecto Aprender es crecer en conexión). Anaya. \\
\hline ESO5 & Romero, R., Ocaña, J., y Mejía D., (2016). ESO 2 Matemáticas (Proyecto somoslink). Edelvives. \\
\hline ESO6 & $\begin{array}{l}\text { Almodóvar, J., Cuadrado A., Díaz, L., Dorce, C., Gámez, J., Marín, S., y Sánchez, D. (2016). Matemáticas Serie } \\
\text { Resuelve ESO } 2 \text { (Proyecto Saber Hacer). Santillana. }\end{array}$ \\
\hline
\end{tabular}

